\title{
Error control in FORM reliability analysis
}

\author{
Laurent Gallimard* \\ LEME, Université Paris Ouest Nanterre La Défense 50 rue de Sèvres, 92410 Ville d'Avray,France
}

\begin{abstract}
This paper deals with the failure probability error induced by the coupling between the first-order reliability method (FORM) and the Finite Element Method (FEM). A FEM error estimator based on the concept of error in constitutive relation associated with goal-oriented error estimation is proposed. Furthermore an importance sampling technique is used to compute the error due to the FORM approximation. Both these errors are used to choose a finite element mesh adapted to the problem.
\end{abstract}

Cet article concerne l'erreur sur la probabilité de défaillance engendrée par le couplage d'une méthode de fiabilité du premier ordre (FORM) avec la méthode des élément finis lors de l'analyse fiabiliste d'une structure élastique. Une technique d'estimation d'erreur en quantité d'intérêt est utilisée pour calculer l'erreur due á l'utilisation d'un discretisation élément finis et une méthode de tirage d'importance pour estimer l'erreur due á l'approximation FORM. Ces deux erreurs sont utilisées pour définir un maillage suffisament raffiné.

Keywords: failure probability; finite element method; FORM approximation; goal-oriented error estimation; importance sampling

Mots-clés: probabilité de défaillance; méthode des éléments finis; approximation FORM; erreur sur des quantités d'intérêt; tirage d'importance

\section{Introduction}

Many applications in structural analysis require taking into account stochastic properties of material, geometry or loads. Different methods based on the probability theory have been developed to quantify the effect of data uncertainties. First-order reliability method (FORM) (Ditlevsen \& Madsen, 1996; Lemaire, 2005) is commonly used in reliability analysis. It is based on the computation of a reliability index (Hasofer \& Lind, 1974), followed by an estimation of the failure probability. In many cases, the structural response cannot be expressed explicitly and the reliability analysis needs to be coupled with a finite element method (FEM). Moreover, the FEM introduces an approximation error in the computation of the reliability index that depends on the mesh used. The control of this discretisation error is then necessary in order to minimise the computation effort, as well as to assess the quality of the computed reliability index.

Methods have been developed over many years to evaluate the global quality of FEM computations (Babuška \& Oden, 1978; Ladevèze \& Leguillon, 1983; Zienkiewicz \& Zhu, 1987). For linear problems, all of these methods provide a global energy-based estimate of

*Email: laurent.gallimard@u-paris10.fr 
the discretisation error. When the structural design criteria involve particular outputs of the FEM (stresses, displacements, intensity factors, ...) this information is insufficient for dimensioning purposes. The development of error estimators for such quantities was initiated in the 80s (Kelly \& Isles, 1989). Recently, numerous works have been published which provide error estimates and bounds for several quantities of interest (Gallimard, 2006; Gallimard \& Panetier, 2006; Ladevèze, Rougeot, Blanchard, \& Moreau, 1999; Peraire \& Patera, 1998; Prudhomme \& Oden, 1999). However, these works have been developed for deterministic FEM and few researches have been carried out on the verification of probabilistic models (Deb, Babuška, \& Oden, 2001; Gallimard, 2011a; Ladevèze \& Florentin, 2006).

In this paper, we consider the case of a linear elastic structure where the limit-state function $G(\mathbf{X})$ is written as: $G(\mathbf{X})=R(\mathbf{X})-S_{\mathrm{h}}(\mathbf{X})$, where $R(\mathbf{X})$ is the resistance of the structure, $S_{\mathrm{h}}(\mathbf{X})$ is the loading effect evaluated by the FEM, and $\mathbf{X}$ is the vector of random variables. The calculation of the failure probability is performed by the FORM approximation. In such a computation two sources of errors are introduced: an approximation error due to the FORM approximation and a discretisation error due to the computation of $S_{\mathrm{h}}$ by the FEM. As far as we know, only the first point has been addressed in the literature (see Mitteau, 1999). In Gallimard (2011a, 2011b) we have shown that the discretisation error may have an important impact on the computed failure probability. In this paper, we also combine both errors in order to find a finite element discretisation adapted to the problem.

The paper is organised as follows: in Section 2, we present the basis of the reliability analysis. The errors, and bounds computation are stated in Section 3. Section 4 is devoted to an analytical example. Then, in Section 5, a method to control the error in a FEM reliability computation is proposed. Finally, a numerical example presents an application to linear elastic fracture mechanics in Section 6.

\section{Reliability analysis}

\subsection{First order method approximation}

Structural reliability aims at computing the probability of failure of a mechanical structure by accounting for uncertainties arising in a model description (geometry, material properties) or the environmental data (prescribed displacement and external forces). These parameters are modelled by a random vector $\mathbf{X}$ and $f_{\mathbf{X}}(\mathbf{x})$ its probability density function. Let us denote by $\mathbf{u}(\mathbf{x})$ the displacement of the structure, and by $S(\mathbf{u}(\mathbf{X}))$ the loading effect in linear elasticity. The failure domain $\mathcal{D}_{\mathrm{f}}$ is described by the limit-state function $G(\mathbf{X})=R(\mathbf{X})-S(\mathbf{u}(\mathbf{X}))$, with $\mathcal{D}_{\mathrm{f}}=\{\mathbf{x} \mid G(\mathbf{x}) \leq 0\}$. The failure probability $P_{\mathrm{f}}$ is:

$$
P_{\mathrm{f}}=\int_{\mathbf{x} \in \mathcal{D}_{\mathrm{f}}} f_{\mathbf{X}}(\mathbf{x}) d \mathbf{x}
$$

A classical way to evaluate this integral is to use the First order Reliability Method FORM (Ditlevsen \& Madsen, 1996). This method is based on the computation of the Hasofer Lind reliability index $\beta$, which allows to compute a simple approximation of $P_{\mathrm{f}}$. The first step of the method consists of using an isoprobabilistic transformation $\mathcal{T}$ to transform $\mathbf{X}$ into a set of independent standardised Gaussian random variables Y (Hasofer \& Lind, 1974). The failure probability $P_{\mathrm{f}}$ is then: 


$$
P_{\mathrm{f}}=\int_{\mathbf{y} \in \mathcal{D}} \phi(\mathbf{y}) d \mathbf{y}
$$

where $\phi(\mathbf{y})$ is the n-dimensional standard normal density with independent and identically distributed components, and $\mathcal{D}$ is the failure domain $(\mathcal{D}=\{\mathbf{y} \mid H(\mathbf{y}) \leq 0\}$ with $H(\mathbf{y}) \equiv G\left(\mathcal{T}^{-1}(\mathbf{y})\right)$. The reliability index $\beta$, is defined as the minimum distance between the origin of the space $O$ and the surface of the limit state

$$
\beta=\min _{\mathbf{y} \in \mathcal{D}} \sqrt{\mathbf{y}^{T} \mathbf{y}}
$$

The solution of this problem gives the value of $\beta$ as well as the coordinates $\mathbf{y}_{i}^{*}$ of a point $P^{*}$ in the space, called the design point. The FORM failure probability is given by

$$
P_{\mathrm{FORM}}=\Phi(-\beta)
$$

where $\Phi$ is the cumulative distribution function of a standard Gaussian variable.

\subsection{Error on the failure probability}

The problem (3) is solved by an optimisation algorithm (in this work we use an improved version of the classical HLRF algorithm, the so-called iHLRF algorithm (Lemaire, 2005; Zhang \& Der Kiureghian, 1995), coupled with finite element analyses). Let us denote by $\mathcal{D}_{\mathrm{h}}$ the failure domain associated with the finite element analyses

$$
\mathcal{D}_{\mathrm{h}}=\left\{\mathbf{y} \in \mathbb{R}^{n} \mid R(\mathbf{y})-S\left(\mathbf{u}_{\mathrm{h}}(\mathbf{y})\right) \leq 0\right\}
$$

where $\mathbf{u}_{\mathrm{h}}(\mathbf{y})$ is the displacement of the structure computed by FEM. The failure domain $\mathcal{D}_{\mathrm{h}}$ defines a failure probability $\mathcal{P}_{\mathrm{h}}$ and an approximation of this failure probability $\mathcal{P}_{\mathrm{h}, \mathrm{FORM}}$ is computed by a FORM approximation.

$$
\beta_{\mathrm{h}}=\min _{\mathbf{y} \in \mathcal{D}_{\mathrm{h}}} \sqrt{\mathbf{y}^{\mathrm{T}} \mathbf{y}} \quad \text { and } \quad \mathcal{P}_{\mathrm{FORM}, \mathrm{h}}=\Phi\left(-\beta_{\mathrm{h}}\right)
$$

The error $e_{\mathrm{f}}$ between the theoretical result $\mathcal{P}_{\mathrm{f}}$ and the computed result $\mathcal{P}_{\text {FORM,h }}$ can be split into two parts

$$
e_{\mathrm{f}}=\mathcal{P}_{\mathrm{f}}-\mathcal{P}_{\mathrm{FORM}, \mathrm{h}}=e_{\mathrm{FORM}}+e_{\mathrm{FORM}, \mathrm{h}}
$$

where $e_{\mathrm{FORM}}=\left(\mathcal{P}_{\mathrm{f}}-\mathcal{P}_{\mathrm{FORM}}\right)$, is the error introduced by the FORM approximation and $e_{\mathrm{FORM}, \mathrm{h}}=\left(\mathcal{P}_{\mathrm{FORM}}-\mathcal{P}_{\mathrm{FORM}, \mathrm{h}}\right)$, is the error introduced by the finite element analyses.

\section{Error analysis}

\subsection{Bounds of the finite element results}

In this section, an elastic structure is considered, and the loading effect is assumed to be a linear functional of the solution $S(\mathbf{u}(\mathbf{Y})$ ) (Gallimard \& Panetier, 2006; Ladevèze et al., 1999; Peraire \& Patera, 1998; Prudhomme \& Oden, 1999). Therefore we can write 


$$
S(\mathbf{u}(\mathbf{Y}))-S\left(\mathbf{u}_{\mathrm{h}}(\mathbf{Y})\right)=S\left(\mathbf{u}(\mathbf{Y})-u_{\mathrm{h}}(\mathbf{Y})\right)
$$

For a given realisation $\mathbf{y} \in \mathbb{R}^{n}$ of the random variables $\mathbf{Y}$, we use an error in the constitutive relation (Ladevèze \& Leguillon, 1983) associated with a method to build admissible stresses based on the partition of unity (Gallimard, 2009) to build lower and upper bounds of $S(\mathbf{u}(\mathbf{y}))$. These bounds depend on the data and on the finite element solution $u_{\mathrm{h}}(\mathbf{Y})$

$$
S^{-}\left(\mathbf{u}_{\mathrm{h}}(\mathbf{y})\right) \leq S(\mathbf{u}(\mathbf{y})) \leq S^{+}\left(\mathbf{u}_{\mathrm{h}}(\mathbf{y})\right) \quad \forall \mathbf{y} \in \mathbb{R}^{n}
$$

The reliability index $\beta_{\mathrm{h}}^{\text {low }}$ associated with the upper bound of the loading effect is

$$
\beta_{\mathrm{h}}^{\text {low }}=\min _{\mathbf{y} \in \mathcal{D}_{\mathrm{h}}^{+}} \sqrt{\mathbf{y}^{T} \mathbf{y}} \quad \text { with } \quad \mathcal{D}_{\mathrm{h}}^{+}=\left\{\mathbf{y} \in \mathbb{R}^{n} \mid R(\mathbf{y})-S^{+}\left(\mathbf{u}_{\mathrm{h}}(\mathbf{y})\right) \leq 0\right\}
$$

The reliability index $\beta_{\mathrm{h}}^{\text {upp }}$ associated with the lower bound of the loading effect is

$$
\beta_{\mathrm{h}}^{\text {upp }}=\min _{\mathbf{y} \in \mathcal{D}_{\mathrm{h}}^{-}} \sqrt{\mathbf{y}^{T} \mathbf{y}} \quad \text { with } \quad \mathcal{D}_{\mathrm{h}}^{-}=\left\{\mathbf{y} \in \mathbb{R}^{n} \mid R(\mathbf{y})-S^{-}\left(\mathbf{u}_{\mathrm{h}}(\mathbf{y})\right) \leq 0\right\}
$$

As $\mathcal{D}_{\mathrm{h}}^{-} \subset \mathcal{D} \subset \mathcal{D}_{\mathrm{h}}^{+}$, the relations (3), (10), (11) lead to the following bounds for the reliability index

$$
\beta_{\mathrm{h}}^{\text {low }} \leq \beta \leq \beta_{\mathrm{h}}^{\text {upp }}
$$

As $\Phi(-\beta)$ is a monotonous function, the computation of a lower and an upper bounds for the reliability index leads to simple bounds of the FORM failure probability due to the finite element approximation.

$$
\mathcal{P}_{\text {FORM }, \mathrm{h}}^{\text {low }} \leq \mathcal{P}_{\text {FORM }} \leq \mathcal{P}_{\text {FORM }, \mathrm{h}}^{\text {upp }}
$$

where $\mathcal{P}_{\mathrm{FORM}, \mathrm{h}}^{\text {low }}=\Phi\left(-\beta_{\mathrm{h}}^{\text {upp }}\right)$ and $\mathcal{P}_{\mathrm{FORM}, \mathrm{h}}^{\text {upp }}=\Phi\left(-\beta_{\mathrm{h}}^{\text {low }}\right)$. The bounds $\beta_{\mathrm{h}}^{\text {low }}$ and $\beta_{\mathrm{h}}^{\text {upp }}$ are computed by solving successively two minimisation problems: (10) and (11). These minimisation problems are solved by the iHLRF algorithm (Zhang \& Der Kiureghian, 1995). At each step, the optimisation algorithm requires the construction of an upper bound $S^{+}\left(\mathbf{u}_{\mathrm{h}}(\mathbf{y})\right.$ ) (or a lower bound $\left.S^{-}\left(\mathbf{u}_{\mathrm{h}}(\mathbf{y})\right)\right)$ of $S(\mathbf{u}(\mathbf{y}))$. In this study, we focus on the errors introduced by the finite element analysis and by the FORM approximation and we assume that the optimisation problems (10) and (11) are solved exactly (see Haukaas \& Der Kiureghian (2006) for detailed studies on the convergence of the optimisation problem). Equation (13) shows that the error introduced by the finite element approximation is bounded by

$$
\left|e_{\mathrm{FORM}, \mathrm{h}}\right| \leq \varepsilon_{\mathrm{FORM}, \mathrm{h}}
$$

with

$$
\varepsilon_{\mathrm{FORM}, \mathrm{h}}=\max \left(\left|\mathcal{P}_{\mathrm{FORM}, \mathrm{h}}^{\mathrm{upp}}-\mathcal{P}_{\mathrm{FORM}, \mathrm{h}}\right|,\left|\mathcal{P}_{\mathrm{FORM}, \mathrm{h}}^{\text {low }}-\mathcal{P}_{\mathrm{FORM}, \mathrm{h}}\right|\right)
$$

\subsection{Error on the FORM approximation}

As far as we know there are few works (Mitteau, 1999) that deal with the estimation of the error on the FORM approximation. A simple method to assess the quality of the 
FORM approximation is the importance sampling (IS) method (Melchers, 1989). The idea is to carry out Monte Carlo simulation with samples having a higher rate of falling in the failure region, because only these samples contribute to the evaluation of $\mathcal{P}_{\mathrm{f}}$. If we denote by $n$ the number of random variables in $\mathbf{Y}$, the integral is first written in the following form:

$$
\mathcal{P}=\int_{\mathbf{y} \in \mathbb{R}^{n}} I(\mathbf{y}) \phi(\mathbf{y}) d \mathbf{y}=\int_{\mathbf{y} \in \mathbb{R}^{n}} \frac{I(\mathbf{y}) \phi(\mathbf{y})}{\psi(\mathbf{y})} \psi(\mathbf{y}) d \mathbf{y}
$$

where $I(\mathbf{y})=1$ if $\mathbf{y} \in \mathcal{D}_{\mathrm{f}}$ and $I(\mathbf{y})=0$ otherwise, is the indicator function and $\psi(\mathbf{y})$ is an IS density. The integral (16) is then computed by a Monte Carlo simulation that leads to an estimation of the probability of failure $P_{\mathrm{f}}$ defined by

$$
\tilde{\mathcal{P}}_{\mathrm{f}}=\frac{1}{N} \sum_{k=1}^{N} \frac{I\left(\mathbf{y}^{k}\right) \phi\left(\mathbf{y}^{k}\right)}{\psi\left(\mathbf{y}^{k}\right)}
$$

where the samples $\mathbf{y}^{k}, k=1, \ldots, N$ are simulated according to $\psi$. The choice of the IS density is an important factor of the method. In our case we choose a multi-normal distribution centred on the design point $P^{*}$ (defined by relation (3)) and a variance 1 . The confidence interval is estimated in a classical way by computing the variance of the samples $s^{2}$. For a $95 \%$ confidence interval we have

$$
\tilde{\mathcal{P}}_{\mathrm{f}}-\Delta(s) \leq \mathcal{P}_{\mathrm{f}} \leq \tilde{\mathcal{P}}_{\mathrm{f}}+\Delta(s)
$$

where $\Delta(s)=1.96 s / \sqrt{N-1}$. Introducing $\tilde{e}_{\mathrm{FORM}}=\tilde{\mathcal{P}}_{\mathrm{f}}-\mathcal{P}_{\mathrm{FORM}}$ and $e_{\mathrm{FORM}}=\mathcal{P}_{\mathrm{f}}-\mathcal{P}_{\mathrm{FORM}}$ in relation (18) leads to

$$
\tilde{e}_{\mathrm{FORM}}-\Delta(s) \leq e_{\mathrm{FORM}} \leq \tilde{e}_{\mathrm{FORM}}+\Delta(s)
$$

In practise the computations are performed with finite element analyses and $I(\mathbf{y})$ is replaced by $I_{\mathrm{h}}(\mathbf{y})$ (where $I_{\mathrm{h}}(\mathbf{y})=1$ if $\mathbf{y} \in \mathcal{D}_{\mathrm{h}}$ and $I_{\mathrm{h}}(\mathbf{y})=0$ otherwise), while $P^{*}$ is replaced by the design point obtained by the resolution of (6). This leads to an approximated value $\tilde{\mathcal{P}}_{\mathrm{f}, \mathrm{h}}$ associated with a variance $s_{\mathrm{h}}^{2}$, and to an approximated value of the bounds

$$
\tilde{e}_{\mathrm{FORM}} \pm \Delta(s) \approx \tilde{e}_{\mathrm{FORM}, \mathrm{h}} \pm \Delta\left(s_{\mathrm{h}}\right) \quad \text { with } \quad \tilde{e}_{\mathrm{FORM}, \mathrm{h}}=\tilde{\mathcal{P}}_{\mathrm{f}, \mathrm{h}}-\mathcal{P}_{\mathrm{FORM}, \mathrm{h}}
$$

An error indicator on the FORM approximation is then defined by

$$
\varepsilon_{\mathrm{FORM}}=\max \left(\left|\tilde{e}_{\mathrm{FORM}, \mathrm{h}}+\Delta\left(s_{\mathrm{h}}\right)\right|,\left|\tilde{e}_{\mathrm{FORM}, \mathrm{h}}-\Delta\left(s_{\mathrm{h}}\right)\right|\right)
$$

Remark: It must be noticed that the bounds proposed in Equation (18) are based on the assumption that the design point is unique. When multiple design points exist, the problem is much more difficult, and as far as we know there is no certain estimate of the error of the FORM approximation (Der Kiureghian \& Dakessian, 1998). Nevertheless, the proposed approach gives a simple error indicator for the FORM approximation, and can be used to define a target error for the error introduced by the FE approximation on the computation of $P_{\mathrm{FORM}}$ as shown in Section 5. 


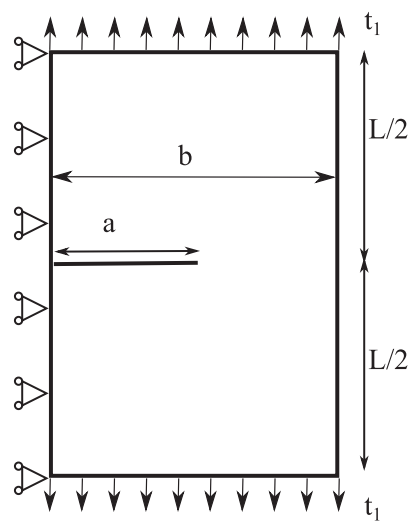

Figure 1. Cracked plate.

\section{An analytical example}

Let us consider the 2D cracked structure $\Omega$ (Figure 1). The problem can be formulated as: Find a displacement field $\mathbf{u}$ and a stress field $\sigma$ defined in $\Omega$ which verify:

$$
\begin{gathered}
\operatorname{div} \boldsymbol{\sigma}=\mathbf{0} \text { and } \boldsymbol{\sigma}=\mathbf{K} \boldsymbol{\varepsilon}(\mathbf{u}) \text { in } \Omega \\
\boldsymbol{\sigma} \cdot \mathbf{n}=\mathbf{T} \quad \text { on } \quad \Gamma_{\mathrm{N}} \text { and } \mathbf{u}=\mathbf{u}_{\mathrm{d}} \text { on } \Gamma_{\mathrm{D}}
\end{gathered}
$$

The external actions on the structure are represented by a prescribed displacement $\mathbf{u}_{\mathrm{d}}$ over a subset $\Gamma_{\mathrm{D}}$ of the boundary and a surface force density $\mathbf{T}$ defined over $\Gamma_{\mathrm{N}}=\partial \Omega-\Gamma_{\mathrm{D}}$. The Hooke's operator of the material is denoted by $\mathbf{K}$. The limit-state of the structure is given by a failure criterion which is the risk that the stress intensity factor $K_{\mathrm{I}}$ reaches the toughness of the material $K_{\mathrm{c}}$.

$$
G=K_{\mathrm{c}}-K_{\mathrm{I}} \leq 0
$$

The deterministic parameters of the model are the young modulus $E=210 \mathrm{GPa}$, the Poisson ratio $v=0.25, L=32 \mathrm{~mm}, b=8 \mathrm{~mm}$. The random variables of the model are the crack length $a$, the toughness of the material $K_{\mathrm{c}}$ and the loading $t_{1}$ as defined in Table 1 . These variables are easily transformed in standard normal variables. The calculation of the $K_{\mathrm{I}}$ consists of an integral around an arbitrary crown $\omega_{2}$ around the tip of the crack

$$
K_{\mathrm{I}}=S(\mathbf{u})=\int_{\omega_{2}}\left(\mathbf{K} \boldsymbol{\varepsilon}\left(\phi \widetilde{\mathbf{u}}^{I}\right)-\phi \tilde{\boldsymbol{\sigma}}^{I}\right): \mathbf{e}(\mathbf{u}) d \omega-\int_{\omega_{2}} \tilde{\boldsymbol{\sigma}}^{I} \operatorname{grad}(\phi) \cdot \mathbf{u} d \omega
$$

where $\tilde{\boldsymbol{\sigma}}^{I}$ and $\tilde{\mathbf{u}}^{I}$ are singular analytical solutions computed at the crack tip and $\phi$ a function defined on $\omega_{2}$ (for further details see Gallimard \& Panetier, 2006). For this problem, analyti-

Table 1. Statistical properties of random variables.

\begin{tabular}{lllcc}
\hline Random variable & Distribution & Units & Mean & Standard deviation \\
\hline Crack length $(a)$ & Log-normal & $\mathrm{mm}$ & 2 & 0.4 \\
Material toughness $K_{\mathrm{c}}$ & Normal & $\mathrm{MPa} \sqrt{\mathrm{mm}}$ & 4 & 0.4 \\
Tensile load $t_{1}$ & Normal & $\mathrm{MPa}$ & 1 & 0.1 \\
\hline
\end{tabular}


cal results are available for $K_{\mathrm{I}}$ in Tada, Paris, and Irwin (2000) (Equation (25)) with $<0.1 \%$ error:

$$
K_{\mathrm{I}}=\left(1-0.025 \alpha^{2}+0.06 \alpha^{4}\right) \sqrt{\sec \frac{\pi \alpha}{2}} t_{1} \sqrt{\pi a} \quad \text { where } \quad \alpha=a / b
$$

For the reliability computations, the initial values of the standard normal variables $\mathbf{u}_{\mathrm{a}}, \mathbf{u}_{\mathrm{t}}$ and $\mathbf{u}_{\mathrm{K}}$ are set to zero. We can obtain a reference value for $\beta$, using the iHLRF algorithm with $K_{\mathrm{I}}$ computed from Equation (25). At convergence the reliability index is $\beta_{\text {ref }}=2.354$ and the FORM failure probability is $\mathcal{P}_{\text {FORM }}=9.29 \times 10^{-3}$.

The structure is meshed with six-noded quadratic triangular elements. To study the evolution of the bounds and the error defined in Section 3.1, a sequence of five uniformly-refined meshes are used. Table 2 show the finite element failure probability $\mathcal{P}_{\text {FORM,h }}$, the computed bounds $\mathcal{P}_{\text {FORM }, \mathrm{h}}^{\text {upp }}, \mathcal{P}_{\text {FORM, }}^{\text {low }}$, the computed error and the effectivity defined as $\rho=\varepsilon_{\mathrm{FORM}, \mathrm{h}} /\left|\mathcal{P}_{\mathrm{FORM}, \mathrm{h}}-P_{\mathrm{FORM}}\right|$. The fact that the finite element failure probability is outside the bounds is not inconsistent, as Equation (13) only implies that $\mathcal{P}_{\mathrm{FORM}, \mathrm{h}}^{\text {low }}$ and $\mathcal{P}_{\mathrm{FORM}, \mathrm{h}}^{\text {upp }}$ bound

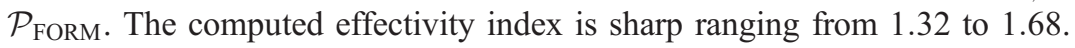

The bounds of the error in the FORM approximation are computed with the IS method proposed in Section 3.2, by using the analytical formula (25), for an increasing number of samples $N$. Table 3 shows the estimated failure probability $\tilde{\mathcal{P}}_{\mathrm{f}}$, the relative range of the confidence interval $r=\Delta(s) / \tilde{\mathcal{P}}_{\mathrm{f}}$ and the error indicator in the FORM approximation $\varepsilon_{\text {FORM }}$. The computed failure probability for $N=10^{5}$ samples can be considered as a reference with $\mathcal{P}_{\mathrm{f}} \approx 1.005 \times 10^{-2}$. As shown in Table 2, a mesh with 670 Degree of Freedom (DoF) is sufficient to lead to a finite element error of the same magnitude than $e_{\mathrm{FORM}}=\mathcal{P}_{\mathrm{f}}-$ $P_{\text {FORM }}=7.6 \times 10^{-4}$.

When the reliability analysis is coupled with the FEM, we use few samples to compute an error indicator on the FORM approximation. Table 4 shows the numerical results corresponding to the computation of the error indicator on the FORM approximation with a set of $N=500$ samples, for a sequence of five uniformly-refined meshes.

Table 2. Bounds of the error introduced by the FEM $-P_{\text {FORM }}=9.29 \times 10^{-3}$.

\begin{tabular}{llllll}
\hline DoF & $\mathcal{P}_{\text {FORM }, \mathrm{h}}$ & $\mathcal{P}_{\mathrm{FORM}, \mathrm{h}}^{\text {low }}$ & $\mathcal{P}_{\mathrm{FORM}, \mathrm{h}}^{\text {upp }}$ & $\varepsilon_{\text {FORM }, \mathrm{h}}$ & $\rho$ \\
\hline 234 & $5.69 \times 10^{-3}$ & $6.04 \times 10^{-3}$ & $1.26 \times 10^{-2}$ & $6.9 \times 10^{-3}$ & 1.68 \\
326 & $7.09 \times 10^{-3}$ & $7.82 \times 10^{-3}$ & $1.00 \times 10^{-2}$ & $2.9 \times 10^{-3}$ & 1.32 \\
670 & $8.54 \times 10^{-3}$ & $8.82 \times 10^{-3}$ & $9.51 \times 10^{-3}$ & $9.6 \times 10^{-4}$ & 1.40 \\
1250 & $8.72 \times 10^{-3}$ & $8.99 \times 10^{-3}$ & $9.42 \times 10^{-3}$ & $6.9 \times 10^{-4}$ & 1.37 \\
1846 & $8.84 \times 10^{-3}$ & $9.13 \times 10^{-3}$ & $9.38 \times 10^{-3}$ & $4.3 \times 10^{-4}$ & 1.56 \\
\hline
\end{tabular}

Table 3. Error indicator on the FORM approximation.

\begin{tabular}{llll}
\hline$N$ & $\tilde{\mathcal{P}}_{\mathrm{f}}$ & $r(\%)$ & $\varepsilon_{\text {FORM }}$ \\
\hline 100 & $8.69 \times 10^{-3}$ & 0.39 & $3.9 \times 10^{-3}$ \\
500 & $1.025 \times 10^{-2}$ & 0.14 & $2.5 \times 10^{-3}$ \\
$10^{3}$ & $1.012 \times 10^{-2}$ & 0.099 & $1.9 \times 10^{-3}$ \\
$10^{4}$ & $1.014 \times 10^{-2}$ & 0.032 & $1.3 \times 10^{-3}$ \\
$10^{5}$ & $1.005 \times 10^{-2}$ & 0.010 & $9.2 \times 10^{-4}$ \\
\hline
\end{tabular}


Table 4. Error indicator on the FORM approximation for 500 samples.

\begin{tabular}{llll}
\hline DoF & $\tilde{\mathcal{P}}_{f, h}$ & $r(\%)$ & $\varepsilon_{\text {FORM }}$ \\
\hline 234 & $6.43 \times 10^{-3}$ & 0.155 & $1.7 \times 10^{-3}$ \\
326 & $7.00 \times 10^{-3}$ & 0.162 & $1.2 \times 10^{-3}$ \\
670 & $9.34 \times 10^{-3}$ & 0.140 & $2.1 \times 10^{-3}$ \\
1250 & $9.44 \times 10^{-3}$ & 0.153 & $2.2 \times 10^{-3}$ \\
1846 & $10.5 \times 10^{-3}$ & 0.114 & $2.8 \times 10^{-3}$ \\
\hline
\end{tabular}

\section{Error control}

As shown in Gallimard (2011a), the precision of the finite element analyses has a great influence on the computed failure probability's error. The control of the quantity $\varepsilon_{\mathrm{FORM}, \mathrm{h}}$ can be achieved by using the techniques developped for goal-oriented mesh adaptivity (Diez \& Calderon, 2007) applied to the control on the error on the loading effect $S(\mathbf{u}(\mathbf{y}))$. The error estimate is required to decide if the meshes used must be refined in order to obtain good approximation of the failure probability and the choice of the prescribed error (a small prescribed error leads to a very refined mesh) will affect the cost of the reliability analysis. Moreover, the choice of the prescribed error is linked with the quality of the FORM approximation. It is not necessary to precisely compute the FORM failure probability, if the FORM approximation is relatively rough.

As shown in Figure $2, \mathcal{P}_{\mathrm{f}}$ can be any value in $\left[\mathcal{P}_{\mathrm{FORM}}-\varepsilon_{\mathrm{FORM}}, \mathcal{P}_{\mathrm{FORM}}+\varepsilon_{\mathrm{FORM}}\right]$. Building a refined mesh such that $\left.\mathcal{P}_{\text {FORM }, \mathrm{h}} \in\right] \mathcal{P}_{\mathrm{FORM}}-\varepsilon_{\mathrm{FORM}}, \mathcal{P}_{\mathrm{FORM}}+\varepsilon_{\mathrm{FORM}}[$ will not give new information on the exact failure probability $\mathcal{P}_{\mathrm{f}}$. So it is reasonable to choose a target error for the finite element approximation greater than $\varepsilon_{\mathrm{FORM}}$. The reliability analysis is then decomposed in three steps:

- Computation of an error indicator on the FORM approximation $\varepsilon_{\mathrm{FORM}}$ with a rough finite element approximation.

- Choice of an admissible target error for the finite element approximation (i.e. $\left.\varepsilon_{\mathrm{FORM}, \mathrm{h}} \geq \varepsilon_{\mathrm{FORM}}\right)$.

- Refinement of the initial mesh (uniformly or with a goal-oriented mesh adaptation (Diez $\&$ Calderon, 2007) until the computed error is smaller than the target error.

\section{Numerical example}

Let us consider the cracked structure shown in Figure 3 and a limit-state given by the failure criterion defined in (23). $K_{\mathrm{I}}$ is computed from the finite element solution with the formula given in Equation (24). The deterministic parameters of the model are the Young's modulus $E=210 \mathrm{GPa}$, the Poisson's ratio $v=0.25, L=20 \mathrm{~mm}, w=8 \mathrm{~mm}$. The random variables

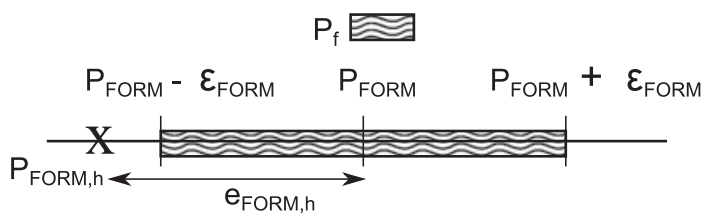

Figure 2. Error on the failure probability. 


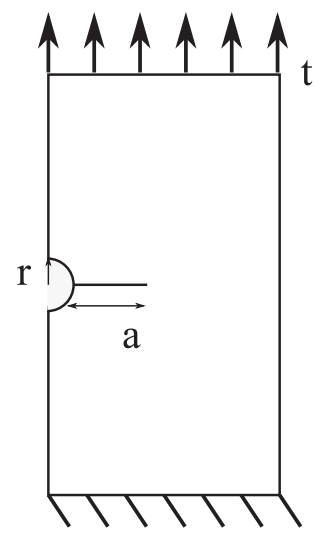

Figure 3. Clamped structure with a crack.

are the crack length $a$, the radius of the circle $r$, the toughness of the material $K_{\mathrm{c}}$ and the load $t$ which are given in Table 5 .

The structure is meshed with six-noded quadratic elements. A sequence of four uniformly refined meshes are used in the analyse (Figure 4). The finite element failure probability $\mathcal{P}_{\text {FORM }, \mathrm{h}}$, the bounds $\mathcal{P}_{\mathrm{FORM}, \mathrm{h}}^{\text {upp }} \mathcal{P}_{\mathrm{FORM}, \mathrm{h}}^{\text {low }}$ and $\varepsilon_{\mathrm{FORM}, \mathrm{h}}$ are shown in Table 6.

The error indicator on the FORM approximation is computed with 500 samples on the initial mesh with $362 \mathrm{DoF}$ and we obtain $\varepsilon_{\mathrm{FORM}}=3.97 \times 10^{-6}$. Figure 5 displays the evolution of the error estimator on the finite element approximation $\varepsilon_{\mathrm{FORM}, \mathrm{h}}$, and shows that the mesh with 1002 DoF is sufficient to perform the reliability analysis. On this mesh the FORM failure probability is $P_{\mathrm{FORM}, \mathrm{h}}=2.97 \times 10^{-5}$, and the computation $\varepsilon_{\mathrm{FORM}, \mathrm{h}} / P_{\mathrm{FORM}, \mathrm{h}}$ leads to a relative error of $19 \%$, which is entirely satisfactory in the case of measurement of reliability based on the FORM approximation.

Table 5. Statistical properties of random variables.

\begin{tabular}{lllrc}
\hline Random variable & Distribution & Units & Mean & Standard deviation \\
\hline Crack length $(a)$ & Log-normal & $\mathrm{mm}$ & 1 & 0.2 \\
Radius $r$ & Log-normal & $\mathrm{mm}$ & 1 & 0.1 \\
Material toughness $K_{c}$ & Normal & $\mathrm{MPa} \sqrt{\mathrm{mm}}$ & 45 & 5 \\
Tensile load $t$ & Normal & $\mathrm{MPa}$ & 250 & 25 \\
\hline
\end{tabular}

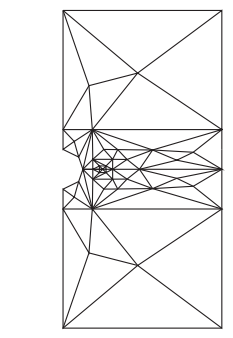

$362 \mathrm{DoF}$

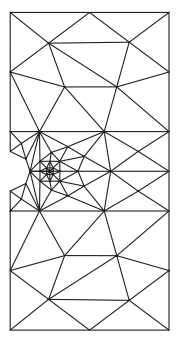

$454 \mathrm{DoF}$

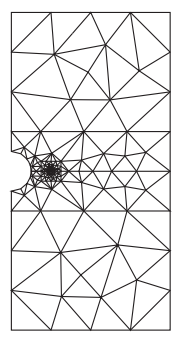

$1002 \mathrm{DoF}$

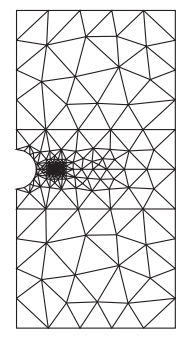

$1910 \mathrm{DoF}$

Figure 4. Clamped structure with a crack: sequence of four meshes. 
Table 6. Failure probability bounds.

\begin{tabular}{llllr}
\hline DoF & $\mathcal{P}_{\text {FORM }, \mathrm{h}}$ & $\mathcal{P}_{\mathrm{FORM}, \mathrm{h}}^{\text {low }}$ & $\mathcal{P}_{\mathrm{FORM}, \mathrm{h}}^{\text {upp }}$ & $\varepsilon_{\mathrm{FORM}, \mathrm{h}}$ \\
\hline 362 & $1.06 \times 10^{-5}$ & $6.02 \times 10^{-6}$ & $7.96 \times 10^{-5}$ & $6.90 \times 10^{-5}$ \\
454 & $2.63 \times 10^{-5}$ & $2.09 \times 10^{-5}$ & $4.31 \times 10^{-5}$ & $1.69 \times 10^{-5}$ \\
1002 & $2.97 \times 10^{-5}$ & $2.61 \times 10^{-5}$ & $3.53 \times 10^{-5}$ & $5.64 \times 10^{-6}$ \\
1910 & $3.14 \times 10^{-5}$ & $3.02 \times 10^{-5}$ & $3.50 \times 10^{-5}$ & $3.60 \times 10^{-6}$ \\
\hline
\end{tabular}

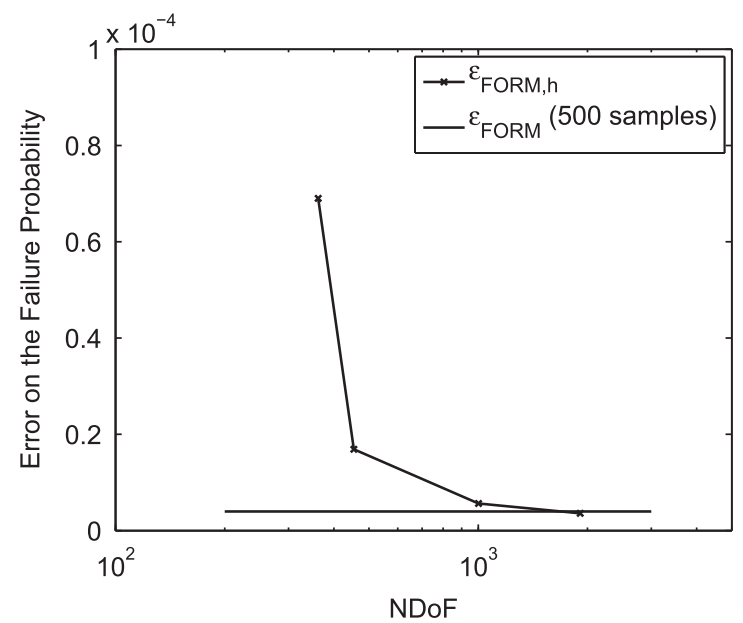

Figure 5. Error estimator on the failure probability.

\section{Conclusion}

A simple method has been presented to recover lower and upper bounds of the FORM failure probability from a standard error in the constitutive relation. An error estimator on the finite element mesh along with an error indicator on the FORM approximation are developed in order to choose an optimal quality for the mesh. These tools will allow to develop goal oriented mesh adaptive strategy during a finite element reliability analysis.

\section{References}

Babuška, I., \& Rheinboldt, W. (1978). A posteriori estimates for the finite element method. International Journal for Numerical Methods in Engineering, 12, 1597-1615.

Deb, M., Babuška, I., \& Oden J. (2001). Solution of stochastics partial differential equations using Galerkin finite element techniques. Computer Methods in Applied Mechanics and Engineering, 190, 6359-6372.

Der Kiureghian, A., \& Dakessian, T. (1998). Multiple design points in first and second-order reliability. Structural Safety, 20, 37-49.

Diez, P., \& Calderon, G. (2007). Remeshing criteria and proper error representations for goal oriented hadaptivity. Computer Methods in Applied Mechanics and Engineering, 196, 719-733.

Ditlevsen, O., \& Madsen, H. (1996). Structural reliability methods. New York, NY: Wiley.

Gallimard, L. (2006). Evaluation of the local quality of the Von Mises's stress and L2-norm of the stress. Engineering Computations, 23(7/8), 876-897.

Gallimard, L. (2009). A constitutive relation error estimator based on traction-free recovery of the equilibrated stress. International Journal for Numerical Methods in Engineering, 78, 460-482.

Gallimard, L. (2011a). Error bounds for the reliability index in finite element reliability analysis. International Journal for Numerical Methods in Engineering, 87, 781-794. 
Gallimard, L. (2011b). Estimation de l'erreur due á la méthode des éléments finis dans une analyse de fiabi-

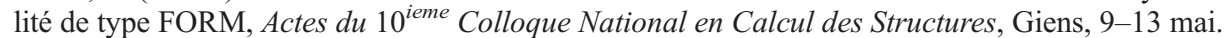

Gallimard, L., \& Panetier, J. (2006). Error estimation of stress intensity factors for mixed-mode crack. International Journal for Numerical Methods in Engineering, 68, 299-316.

Hasofer, A., \& Lind, N. (1974). An exact and invariant first order reliability format. Journal of Engineering Mechanics - ASCE, 100, 111-121.

Haukaas, T., \& Der Kiureghian, A. (2006). Strategies for finding the design point in non-linear finite element reliability analysis. Probabilistic Engineering Mechanics, 21, 133-147.

Kelly, D., \& Isles, J. (1989). Procedures for residual equilibration and local error estimation in the finite element method. Communications in Applied Numerical Methods, 5, 497-505.

Ladevèze, P., \& Florentin, E. (2006). Verification of stochastic models in uncertain environments using the constitutive relation error method. Computer Methods in Applied Mechanics and Engineering, 196, 225-234.

Ladevèze, P., \& Leguillon, D. (1983). Error estimate procedure in the finite element method and application. SIAM Journal on Numerical Analysis, 20(3), 485-509.

Ladevèze, P., Rougeot, P., Blanchard, P., \& Moreau, J. (1999). Local error estimators for finite element analysis. Computer Methods in Applied Mechanics and Engineering, 176, 231-246.

Lemaire, M. (2005). Fiabilité des structures. Paris: Hermes.

Melchers, R. (1989). Importance sampling in structural systems. Structural Safety, 6, 3-10.

Mitteau, J. (1999). Error evaluation for the computation of failure probability in static structural reliability problems. Probabilistic Engineering Mechanics, 14, 119-135.

Peraire, J., \& Patera, A. (1998). Bounds for linear-functional outputs of coercive partial differential equations: local indicators and adaptive refinement. In P. Ladevèze \& J. Oden (Eds.), Advances in adaptive computational methods (pp. 199-216). Cachan: Elsevier.

Prudhomme, S., \& Oden, J. (1999). On goal-oriented error estimation for elliptic problems: application to the control of pointwise errors. Computer Methods in Applied Mechanics and Engineering, 176, 313-331.

Tada, H., Paris, P., \& Irwin, G. (2000). The stress analysis of crack handbook. New York, NY: ASME Press.

Zhang Y., \& Der Kiureghian, A. (1995). Two improved algorithms for reliability analysis. Reliability and Optimization of Structural Systems. In R. Rackwitz, G. Augusti and A. Borri (Eds.) Proceedings 6th IFIP WG7.5 working conference on reliability and optimization of structural systems. (pp. 297-304). Chapman \& Hall.

Zienkiewicz, O., \& Zhu, J. (1987). A simple error estimator and adaptive procedure for practical engineering analysis. International Journal for Numerical Methods in Engineering, 24, 337-357. 